\title{
Composição e estrutura de uma floresta primária atingida por incêndio florestal na Amazônia Oriental
}

\author{
Composition and structure of primary forest affected by forest fire in Eastern \\ Amazon
}

\author{
Dárlison Fernandes Carvalho de Andrade ${ }^{\mathrm{I}}$, Ademir Roberto Ruschel" ${ }^{\mathrm{II}}$, \\ Angela Luciana de Avila' ${ }^{\text {III }}$, João Ricardo Vasconcellos Gama ${ }^{\mathrm{I}}$
}

\begin{abstract}
Resumo
A dinâmica da composição florística de uma área de floresta primária, atingida por fogo, em 1997, na Amazônia brasileira, foi analisada, comparando-se dados de inventários amostrais, realizados nos anos de 1983, 1987, 1989, 1995, 2008 e 2012, em 12 parcelas permanentes de 0,25 hectares, com dados anteriores (14 anos) e posteriores ao incêndio (15 anos). A área de estudo está localizada em uma floresta ombrófila densa, sem histórico de distúrbios antrópicos, na Floresta Nacional do Tapajós, oeste do estado do Pará. As questões deste estudo são as seguintes: (1) Qual o efeito de um primeiro incêndio na composição de espécies arbóreas de uma floresta primária na Amazônia? (2) Existe diferença, na diversidade de espécies arbóreas, antes e após o fogo? Para responder essas questões, (i) foram descritas, quantificadas e comparadas a composição de espécies arbóreas de antes e depois o fogo, (ii) foram quantificadas e comparadas a riqueza e a diversidade de espécies arbóreas de antes e após o fogo. Após 15 anos da ocorrência do incêndio, a floresta não registrou perdas em riqueza e diversidade de espécies arbóreas, indicando boa resiliência ao distúrbio. As alterações pós-fogo na composição florística de uma floresta primária são definidas, principalmente, pela entrada e saída de espécies localmente raras, e as espécies com maior densidade e área basal são responsáveis pela manutenção da estrutura da floresta, destacando-se dentre elas: Rinorea guianensis Aubl. e Protium apiculatum Swart.
\end{abstract}

Palavras-chave: Composição florística; Floresta tropical; Resiliência

\begin{abstract}
The floristic composition dynamics of a primary forest in the Brazilian Amazon, which was affected by fire in 1997, was analyzed by comparing data from sample inventories carried out in 1983, 1987, 1989, 1995, 2008 and 2012 in 12 plots of 0.25 ha each including therefore previous (14 years) and post-fire (15 years) periods. The study area is located in an area of dense ombrophilous forest, unaffected by anthropogenic disturbances, in the Tapajós National Forest, Pará state, Brazil. The questions of this study are the following: (1) What is the effect of a fire on the tree species composition of a mature forest in the Amazon? (2) Is there a difference in the diversity of tree species between before and after fire? To answer these questions, (i) the pre-and post-fire tree species composition were described, quantified and compared, and (ii) the richness and diversity of pre-and post-fire tree species were quantified and compared. After 15 years of fire, the forest had no loss in richness and diversity of tree species, indicating good resilience to the fire disturbance. The post-fire changes in the floristic composition of this primary forest are mainly driven by the gain and loss of locally rare species. Furthermore, some tree species with higher density and basal area were responsible for the maintenance of the forest structure, especially Rinorea guianensis Aubl. and Protium apiculatum Swart.
\end{abstract}

Keywords: Floristic composition; Tropical forest; Resilience

\footnotetext{
Engenheiro Florestal, MSc./Dr., Aluno/Professor, Programa de Pós-Graduação em Sociedade, Natureza e Desenvolvimento, Universidade Federal do Oeste do Pará, Avenida Mendonça Furtado, 2946, Fatima, CEP: 68040-470, Santarém (PA), Brasil. darlisonicmbio@gmail.com (ORCID: 00000002-4362-8979) / jrvgama@gmail.com (ORCID: 0000-0002-3629-3437)

II Engenheiro Agrônomo, Dr., Embrapa Amazônia Oriental, Tv. Dr. Enéas Pinheiro, s/n, Marco, CEP 66095-903, Belém (PA), Brasil. ademir.ruschel@ embrapa.br (ORCID: 0000-0002-0352-5238)

III Forest Engineer, Dr ${ }^{\mathrm{a}}$., Researcher at Forest Research Institute of Baden-Wuerttemberg/FVA, Department of Forest Growth, Wonnhaldestraße 4, 79100, Freiburg, Germany. angeladeavila@gmail.com (ORCID: 0000-0001-8291-7936)
} 


\section{Introdução}

Desde o último terço do século XX, com as políticas de ocupação da Amazônia e a expansão do agronegócio, a riqueza dos ecossistemas florestais desta região tem sido ameaçada pelas drásticas mudanças ocorridas na paisagem. No Estado do Pará, a construção de rodovias, a extração seletiva de madeira, a mineração e a agropecuária provocaram a fragmentação da vegetação e, estas atividades de uso da terra, associadas a eventos climáticos como El Niño, têm contribuído para o aumento dos incêndios na região (HOLDSWORTH; UHL, 1997; BARLOW et al. 2004). Somente no Pará, que representa, aproximadamente, $25 \%$ de toda a Amazônia Brasileira, o quantitativo de área de floresta alterada, entre os anos de 2006 a 2015, por incêndio ou extrações seletivas de madeira, é maior do que a área de floresta desmatada em toda a Amazônia Brasileira, no mesmo período (BARLOW et al., 2016).

Incêndios florestais ocorriam na floresta Amazônica com grandes intervalos de recorrência, os quais já foram de 400 - 700 anos (MEGGERS, 1994), e atualmente não passam de décadas. Os efeitos combinados de El Niño e as mudanças de uso da terra têm aumentado drasticamente a frequência do fogo em florestas tropicais úmidas (BARLOW et al., 2016). Os eventos de fogo ou a extração seletiva de madeira na Amazônia são distúrbios com duração, intensidade e frequência variável, ocorrem de forma abrupta e podem alterar de forma significativa a diversidade de espécies no bioma (KARSTEN et al., 2013).

$\mathrm{Na}$ floresta amazônica há uma elevada ocorrência de espécies raras (ANDRADE et al., 2015; OLIVEIRA et al., 2005) e poucas espécies com alta densidade (STEEGE et al., 2013), característica que potencializa os riscos de ações humanas levarem a extinção de espécies com baixa abundância e mais suscetíveis a alterações ambientais. No estudo de Araujo et al. (2013) em uma floresta primária do Acre, incendiada em 2005, a fitocenose apresentou modificações na composição florística com redução na diversidade de espécies, indicando sérios prejuízos estruturais e funcionais ao ecossistema.

A dinâmica das florestas tropicais é contínua e a comunidade vegetal, passa por uma série de estágios de desenvolvimento. A dinâmica da vegetação arbórea, portanto, não cessa quando a floresta atinge um desenvolvimento estrutural máximo (clímax), permanecem os distúrbios localizados (endógenos), como quedas de árvores ou inundações locais, que não afetam de maneira uniforme todo o conjunto da floresta (CHAZDON, 2012).

Ambientes com distúrbios que resultem em grandes clareiras podem beneficiar espécies pioneiras (REIS et al., 2010), mas espécies tolerantes à sombra que, apesar de se desenvolverem em condições de dossel fechado e sub-bosque, beneficiam-se da radiação solar proveniente de clareiras, sejam naturais ou provenientes da exploração florestal, embora este benefício diminua gradativamente à medida que o tempo passa (JARDIM; SOARES, 2010). O processo dinâmico da recomposição de florestas atingidas por distúrbios antrópicos pode ser acompanhado por meio do inventário florestal contínuo, utilizando-se parcelas permanentes (COSTA et al., 2002), nas quais se realizam contagem, medição, posterior recontagem e remedição de plantas sobreviventes.

A dinâmica de uma área de floresta ombrófila densa sem histórico de extração de madeira, e atingida por um incêndio, em 1997, foi analisada, comparando-se dados de monitoramento anterior ao fogo (1983 - 1995) e pós-fogo (1997 - 2012). As questões deste estudo são as seguintes: (1) Qual o efeito de um primeiro incêndio na composição de espécies arbóreas de uma floresta primária na Amazônia? (2) Existe diferença, na diversidade de espécies arbóreas, antes e após o fogo? Para responder essas questões, (i) foram descritas, quantificadas e comparadas a composição de espécies arbóreas de antes e após o fogo e (ii) foram quantificadas e comparadas a riqueza e a diversidade de espécies arbóreas de antes e após o fogo. 


\section{Material e métodos}

\section{Área de estudo}

Aproximadamente 527.000 hectares integram a Floresta Nacional do Tapajós (FLONA do Tapajós), Unidade de Conservação (UC) Federal, localizada no oeste do estado do Pará, às margens da Rodovia BR-163 (ANDRADE et al., 2015). O clima é quente e úmido (Am na classificação Köppen) com precipitação média anual de $2.000 \mathrm{~mm}$, uma estação seca (agosto a novembro) e temperatura média anual de $25^{\circ} \mathrm{C}$. O tipo de solo predominante é um Latossolo Amarelo Distrófico ou Latossolo, com textura argilosa pesada, perfil profundo e baixa fertilidade (OLIVEIRA JUNIOR et al., 2015)

A área de estudo está localizada na região de planalto da FLONA do Tapajós $\left(3^{\circ} 18^{\prime} \mathrm{S} / 3^{\circ} 19^{\prime} \mathrm{S}\right.$, $\left.54^{\circ} 56^{\prime} \mathrm{W} / 54^{\circ} 57^{\prime} \mathrm{W}\right)$, próxima ao $\mathrm{Km} 114$ da Rodovia BR-163, e representava, na época de instalação das parcelas, uma típica floresta primária densa de terra firme da região, sem exploração florestal (CARVALHO, 2002), com dossel uniforme ou com árvores emergentes (GONÇALVES; SANTOS, 2008).

Em 1983 foram selecionados 36 hectares de floresta sem histórico de exploração florestal, nos quais, houve uma subdivisão da área em 4 quadrantes de 9 ha $(300 \mathrm{~m}$ x $300 \mathrm{~m})$, e em cada um deles foram instaladas 3 (três) parcelas de 0,25 hectares $(50 \mathrm{~m} \times 50 \mathrm{~m}$ ), de forma inteiramente ao acaso, totalizando 12 parcelas, sendo cada uma delas subdividida em 25 subparcelas de 10 m x 10 m, numeradas de 1 a 25 para facilitar a localização das árvores e controle das ações nos inventários contínuos (Figura 1).

\section{Figura 1 - Localização das parcelas atingidas e não atingidas pelo fogo na Floresta Nacional do Tapajós, Pará, Amazônia, Brasil}

Figure 1 - Location of the affected and unaffected by fire plots in Tapajós National Forest,

Pará state, Amazon, Brazil

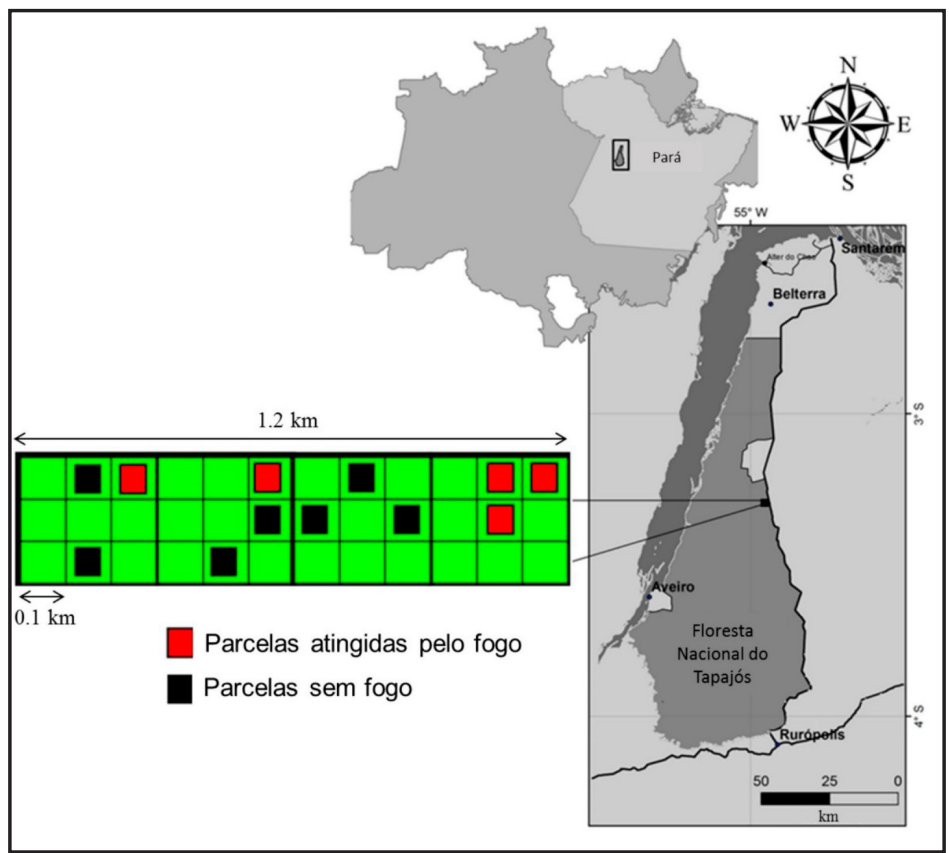

Fonte: Dárlison Fernandes Carvalho de Andrade (2019) 


\section{Base de dados}

Todas as árvores com diâmetro medido a $1,30 \mathrm{~m}$ do solo (DAP) $\geq 5 \mathrm{~cm}$ foram mensuradas em 1983, 1987, 1989, 1995, 2008 e 2012, conforme metodologia descrita em Silva et al. (2005). Todas as árvores foram identificadas com plaquetas de alumínio numeradas (parcela, subparcela e número da árvore) para permitir o acompanhamento de seu crescimento e sobrevivência ao longo dos anos. Todas as variáveis mensuradas nas parcelas foram processadas no software Monitoramento de Florestas Tropicais (MFT) da Embrapa Amazônia Oriental e os dados foram exportados para planilhas eletrônicas para análises gráficas e estatísticas.

Todas as árvores foram identificadas, na floresta, por seus nomes vernaculares ou científicos por parataxonomistas. As árvores não identificadas tiveram o seu material botânico coletado para identificação no herbário da Embrapa Amazônia Oriental. As espécies foram classificadas de acordo com APG IV (2016), e sua identificação pad ronizada conforme classificação do REFLORA (2019).

\section{Identificação das parcelas atingidas pelo fogo}

Em dezembro de 1997, um incêndio acidental de superfície queimou uma faixa de 1.200 metros de extensão nas proximidades do Km 114 da Rodovia BR-163 da FLONA do Tapajós. O fogo atingiu a vegetação arbórea com muita rapidez e uma equipe de funcionários da unidade regional da Embrapa ficou no local durante dois dias até que o fogo fosse apagado, reduzindo-se os danos. Informações detalhadas sobre o aumento da mortalidade e recrutamento de árvores em decorrência deste incêndio podem ser obtidas em Andrade et al. (2019).

O histórico de monitoramento da área indica que metade das 12 parcelas foram atingidas pelo fogo, porém, não há uma definição sobre quais critérios foram utilizados nesta identificação. Por isso, para categorizar as parcelas em T0 (sem fogo) e T1 (queimadas), foram utilizados os dados de mortalidade e recrutamento de árvores nos períodos pós-fogo (1995-2008 e 2008-2012) em uma análise de semelhança entre as parcelas ("cluster"), com uso da função "kmeans" do pacote vegan no software R (LEGENDRE; LEGENDRE, 2012), permitindo a identificação de um padrão comum, entre as parcelas atingidas pelo fogo e aquelas não atingidas, em relação à dinâmica de mortalidade e recrutamento de árvores. Ao final desta análise, foram identificadas cinco parcelas atingidas pelo fogo, denominadas "T1" e sete parcelas "sem fogo", denominadas "T0".

\section{Composição e diversidade florística}

A diversidade foi calculada mediante o emprego do índice de diversidade de Shannon (H') (CONDÉ; TONINI, 2013) e foram comparadas a riqueza de espécies registrada em cada uma das áreas (T0 e T1) com a riqueza estimada pelo método Chao de primeira ordem (Chao 1) que se baseia na abundância das espécies raras. A estimativa de riqueza é calculada pela equação:

$$
\text { Chao } 1=\mathrm{S}_{\mathrm{obs}}+\frac{a^{2}}{2 \mathrm{~b}^{2}}
$$

Em que: "S ${ }_{\text {obs }}$ " o número de espécies observado nas amostras, "a” é o número de espécies representadas por apenas um espécime, e "b" é o número de espécies representado por exatamente dois espécimes (COLWELL; CODDINGTON, 2016).

As respostas na riqueza de espécies foram analisadas pela comparação do acréscimo (ingresso) ou perda de espécies (egresso) desde a primeira medição (1983) até a última, em 2012 (AVILA et al., 2015). Foram consideradas como espécies localmente raras aquelas que possuíam, em cada uma das medições, apenas 1 (uma) árvore representando a espécie, para T0 e T1 (AMARAL et al., 2009). 


\section{Estrutura}

Além da utilização de gráficos de ranking de abundância para avaliar a distribuição das espécies entre as medições para as parcelas T0 e T1 (QUARESMA et al., 2018), os parâmetros fitossociológicos da estrutura horizontal da floresta (frequência, densidade, dominância e valor de importância das espécies) foram calculados conforme definições descritas em Silva et al. (2008).

\section{Análise Estatística}

A diversidade de espécies $\left(\mathrm{H}^{\prime}\right)$ para cada medição foi comparada considerando-se o grau de dependência entre as medições realizadas, ou seja, medição das parcelas atingidas pelo fogo comparada com a medição posterior das parcelas atingidas pelo fogo, e da mesma forma as comparações foram feitas com as parcelas que não foram atingidas pelo fogo (SIT; TAYLOR, 1998). As comparações entre as medições ocorreram por meio de uma Análise de Variância (ANOVA) para medidas repetidas, considerando-se o tempo como parcelas subdivididas, em razão da dependência entre as múltiplas observações nas mesmas unidades de amostra (REIS et al., 2015; VON ENDE, 1993).

Para atender as premissas da ANOVA de medidas repetidas, foram aplicados os testes de normalidade de Shapiro-Wilk e de homogeneidade de variância de Bartlett. O valor de $F$ foi corrigido usando-se os fatores de correção Greenhouse-Geisser (G-G) ou Huynh-Feldt (H-F) para inferir sobre cada fonte de variação (VON ENDE, 1993). Foi utilizado para as inferências, o menor fator (G-G ou H-F) ao invés do valor de "valor de p" para o teste de hipótese:

Hipótese nula $(\mathrm{H} 0) \mu 1=\mu 2=\mu 3$ : Isto é, não há diferença significativa entre os valores do Índice de Diversidade ( $\left.H^{\prime}\right)$ para as medições ou períodos; Hipótese alternativa $(\mathrm{H} 1) \mu 1 \neq \mu 2 \neq \mu 3$ : existe pelo menos uma média $\left(\mathrm{H}^{\prime}\right)$ diferente entre as medições ou períodos.

\section{Resultados e discussão}

\section{Composição e Diversidade florística}

No inventário de $1983,3.360$ árvores com DAP $\geq 5 \mathrm{~cm}$ foram inventariadas, nas 12 parcelas de floresta, equivalentes, em média, a 1.120 árvores ha $\mathrm{h}^{-1} \mathrm{e} 29,98 \mathrm{~m}^{2} \mathrm{ha}^{-1}$ de área basal $(\mathrm{CV}=18,01 \%$; Erro de Amostragem Relativo = 11,44\%). Deste total, foram registradas, em T0, 1.041 árvores ha ${ }^{-1}$ pertencentes a 235 espécies e 52 famílias botânicas e, em T1, 1.230 árvores ha ${ }^{-1}$ de 248 espécies e 52 famílias. As cinco famílias mais abundantes foram as mesmas em T0 e T1, sendo elas: Fabaceae (39 espécies em T0 e, 39, em T1), Sapotaceae (22; 25), Lecythidaceae (13; 13), Moraceae $(13 ; 12)$ e Annonaceae $(10 ; 10)$.

Após 29 anos (2012), 3.260 árvores foram inventariadas nas 12 parcelas, equivalentes a 1.087 árvores ha ${ }^{-1}$ e 32,81 $\mathrm{m}^{2}$ ha-1 de área basal, sendo 1.151 árvores ha ${ }^{-1}$ de 239 espécies e 49 famílias em T0 e 1045 árvores ha ${ }^{-1}$ de 250 espécies e 52 famílias em T1 (Figura 3b). Em T0, as famílias Fabaceae (40), Sapotaceae (22), Lecythidaceae (15), Moraceae (13) permaneceram dominando a área e Annonaceae (9) perdeu posição para Lauraceae (11) entre as 5 famílias com mais espécies. Em T1, as famílias botânicas responsáveis pela maior quantidade de espécies, em 1983, continuaram dominando o local em 2012 - Fabaceae (36), Sapotaceae (26), Moraceae (15), Lecythidaceae (13), e Annonaceae (9). 
Ao longo dos 29 anos de monitoramento, os testes estatísticos não identificaram diferenças significativas entre nenhuma das medições comparadas, portanto, não houve perda de diversidade em T0 e nem em T1. Em T0, o Índice de Diversidade de Shannon variou de 4,15 $(1983 ; 1987 ; 1989)$ a 4,19 (2008; 2012), enquanto em T1 o menor valor também foi registrado em $1983(4,27)$ e o maior em 2008 e 2012 (4,38), ou seja, após o fogo (Figura 2a). Os resultados são condizentes com outros estudos realizados na Floresta Nacional do Tapajós (OLIVEIRA et al., 2005; GONÇALVES; SANTOS et al., 2008) e indicam que a severidade do fogo não foi suficiente para comprometer a capacidade natural que o ecossistema tem para se recuperar do distúrbio, resultado, também, observado por Martins et al. (2012)

Segundo o estimador Chao 1, após o fogo (2008) T1 e T0 seguiram trajetórias diferentes com maior distanciamento entre os valores de riqueza, indicando, provavelmente, maior presença de espécies com baixa densidade, em T1, na medição de 2008 (Figura 2b), considerando-se os parâmetros utilizados por Chao 1 (COLWELL; CODDINGTON, 1994). Comparando-se a riqueza de espécies entre 1983 e 2012, tanto em T0 quanto em T1, verificou-se perdas de espécies, compensadas pela entrada de outras ao longo do tempo, até mesmo no período pós-fogo, em T1, o aumento na saída das espécies foi acompanhado por um aumento proporcional na entrada de novas espécies (Figura 3), indicando um balanço dinâmico na floresta que resultou em saldo positivo na riqueza de espécies observada em T0 e T1 (Figura 2b).

Figura 2 - Índice de diversidade de Shannon $(A)$ e riqueza de espécies $(B)$ em uma área de floresta madura sem histórico de perturbações e em uma área de floresta madura com registro de incêndio, no ano de 1997, na Floresta Nacional do Tapajós, Pará, Amazônia,

\section{Brasil}

Figure 2 - Shannon diversity index (A) and species richness (B) in an area of the mature forest without a history of disturbance (c) and an area of mature forest with fire record, in 1997,

Tapajós National Forest, Pará state, Amazon, Brazil

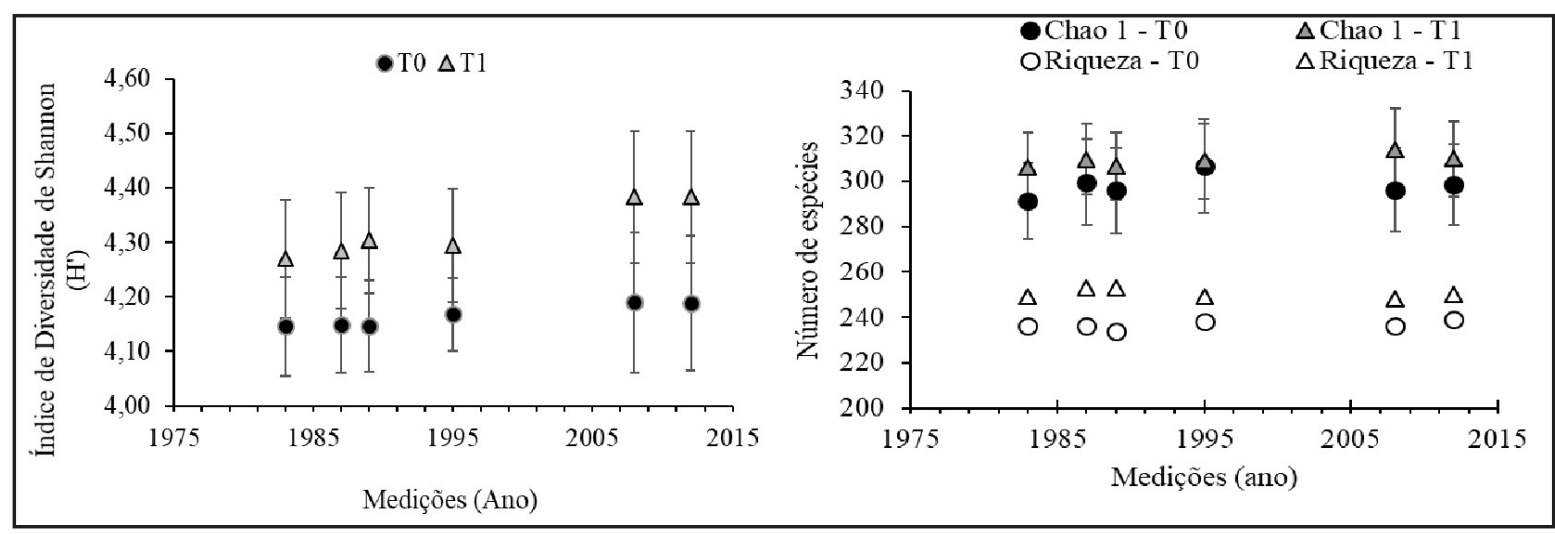

Fonte: Dárlison Fernandes Carvalho de Andrade (2019) 
Figura 3 - Dinâmica de ingresso e egresso de espécies em uma área de floresta madura sem histórico de perturbações (T0) e em uma área de floresta madura com registro de incêndio (T1), no ano de 1997, Floresta Nacional do Tapajós, Pará, Amazônia, Brasil

Figure 3 - Dynamics of entry and exit of species in an area of the mature forest without a history of disturbance (A) and in an area of mature forest affected by fire record, in 1997, Tapajós National Forest, Pará state, Amazon, Brazil

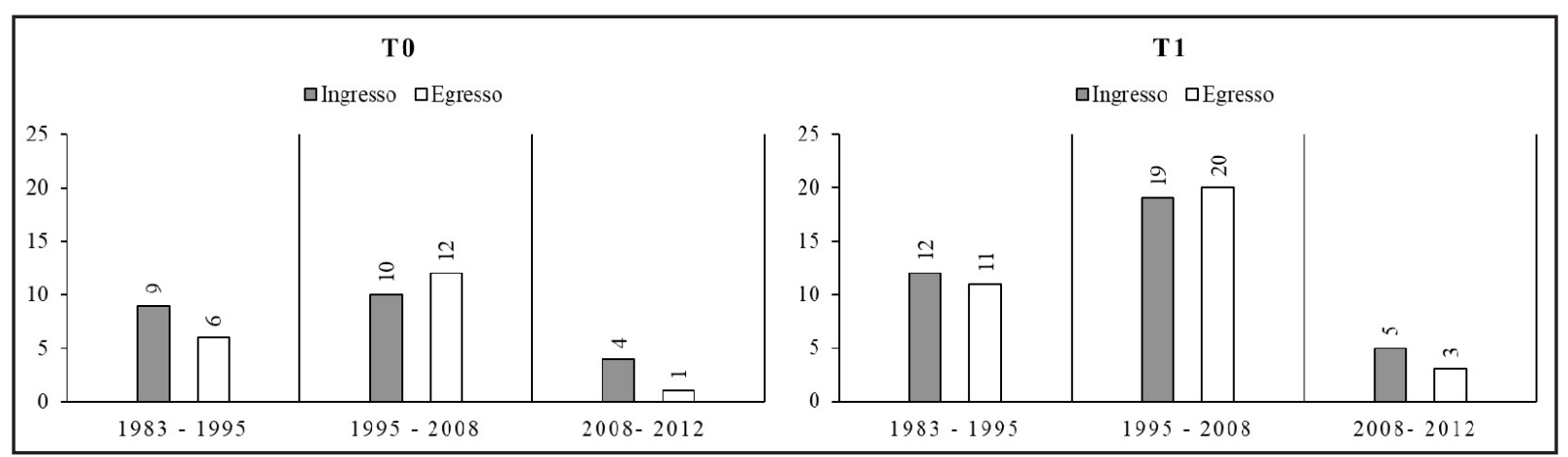

Fonte: Dárlison Fernandes Carvalho de Andrade (2019)

Segundo Oliveira e Amaral (2004), altas taxas de recrutamento de novas árvores pertencentes a espécies localmente raras podem aumentar a dissimilaridade entre comunidades. Esperava-se que a diversidade de árvores diminuísse na floresta atingida por fogo como já relatado por Barlow et al. (2016), mas isso não ocorreu. Há de se ressaltar que o tempo decorrido de 11 (2008) e 15 anos (2012), após a ocorrência do incêndio (1997), parece ter sido suficiente para que a floresta tenha recuperado suas condições de diversidade originais, resultado também encontrado por Martins et al. (2012), em uma floresta ombrófila densa de Roraima, no Brasil, 12 anos após o fogo.

\section{Estrutura}

Em 1983, 69 das 235 espécies registradas em T0 eram localmente raras, ou seja, representadas por apenas 1 árvore amostrada (29,36\%), e da mesma forma, em T1, 84 das 249 espécies possuíam apenas 1 árvore (33,73\%). Segundo Souza et al. (2006), a estrutura de uma floresta ombrófila densa na Amazônia caracteriza-se por alta diversidade florística, poucas espécies dominantes e muitas espécies com baixa densidade. O fogo não alterou esta estrutura em T1 (Figura 4). 


\section{Figura 4 - Ranking de abundância das espécies arbóreas, em T1, comparando a última mediação antes do incêndio (1995) e a primeira medição após o fogo (2008) na Floresta Nacional do Tapajós, Pará, Amazônia, Brasil}

Figure 4 - Ranking of the abundance of tree species in T1, comparing the last measurement before the fire (1995) and the first measurement ater the fire (2008), in Tapajós National Forest,

Pará state, Amazon, Brazil

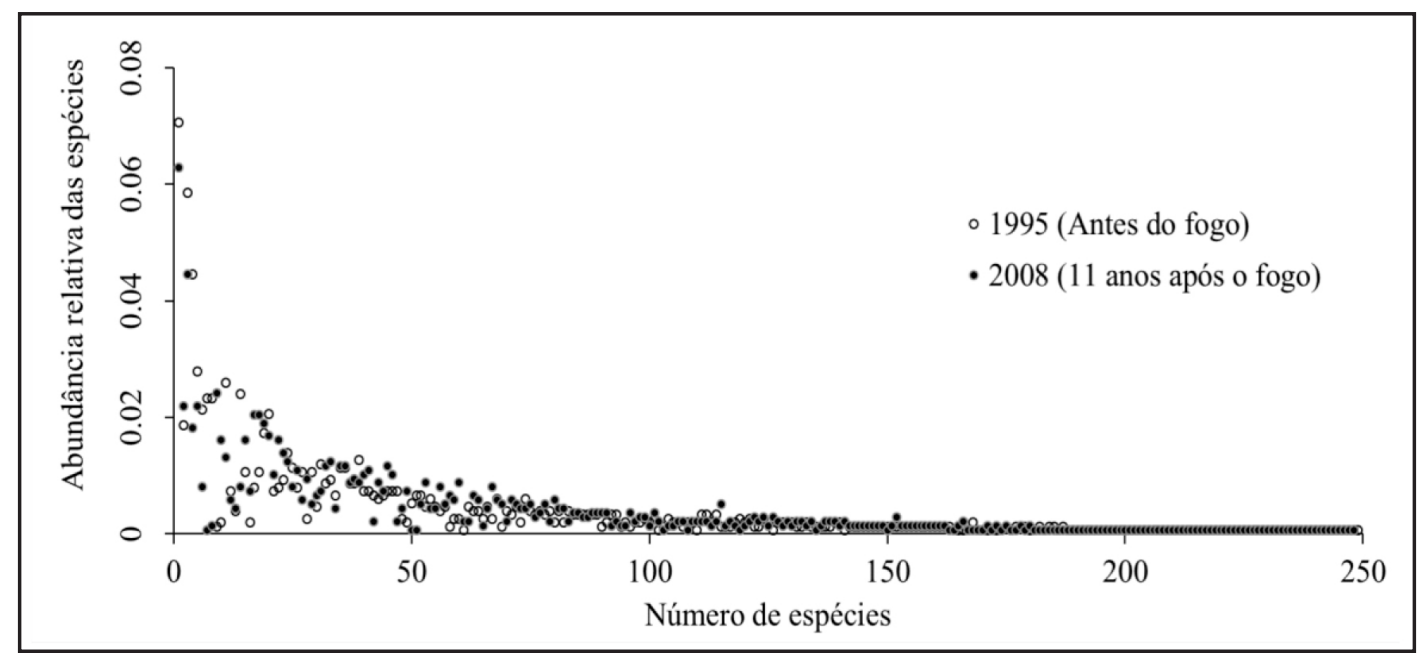

Fonte: Dárlison Fernandes Carvalho de Andrade (2019)

Em relação às espécies com altos valores de densidade absoluta, em 1983, 22,89\% do total de árvores registradas, em T1, eram pertencentes a apenas cinco espécies - Rinorea guianensis, Protium apiculatum, Inga spp., Eschweilera coriacea e Duguetia echinophora - e, em 1995, essas mesmas espécies respondiam por $22,77 \%$ do total de árvores. Em 2012, Cordia spp. passou a ocupar posição de destaque entre aquelas com maior densidade de árvores, ficando atrás apenas de Rinorea guianensis e Protium apiculatum que se mantiveram como as espécies com maior densidade nos 29 anos de monitoramento. Apesar da redução na população das espécies mais abundantes, ao longo dos 29 anos, as cinco espécies - Rinorea guianensis (18,45\% de redução), Protium apiculatum (22,22\% de redução), Inga spp. (58,75\% de redução), Cordia spp. (280\% de aumento) e Neea floribunda (21,05\% de redução) juntas respondiam por $17 \%$ do total de árvores na área.

Em T0, também se repetiram os padrões de dominância por poucas espécies e de maior movimentação de entrada e saída de espécies ao longo do tempo, principalmente, por espécies localmente raras (Figura 3). Em T0, em 1983, houve menor redução na população das cinco espécies mais abundantes, que foram as mesmas registradas em T1 - Rinorea guianensis, Protium apiculatum, Duguetia echinophora, Inga spp. e Eschweilera coriacea. Estas espécies respondiam por 22,67\% do total de árvores, em 1983 - e, em 2012, as cinco espécies mais abundantes respondiam por 20,51\% do total de árvores - Rinorea guianensis, Protium apiculatum, Inga spp., Onychopetalum amazonicum, Duguetia echinophora e Amphiodon effusus, sendo que Rinorea guianensis e Protium apiculatum não tiveram redução de suas populações, mas Duguetia echinophora teve redução de 32,43\%, Inga spp. de 51,39\% e Eschweilera coriacea de 22,22\%.

Nos estudos de Souza et al. (2006), Rinorea guianensis se destacou com altos valores de densidade absoluta e ter Steege et al. (2013) a incluiu, juntamente com Duguetia echinophora, na lista das 20 espécies arbóreas mais abundantes da Amazônia, característica condizente com a presença desta espécie como aquela que manteve a maior população ao longo de 29 anos, tanto em T0 quanto em T1. Protium apiculatum também manteve seu destaque na estruturação da floresta por todo o período de monitoramento, em T0 e T1 (Tabelas 1 e 2). Oliveira e Amaral (2004), também, registraram esta espécie 
com altos valores de densidade absoluta. O fogo, embora tenha reduzido as populações das espécies com maior densidade absoluta, não prejudicou Rinorea guianensis e Protium apiculatum ao ponto de retirá-las da lista das espécies com maiores valores de IVI na comunidade. O comportamento dessas espécies indica que, mesmo em áreas de floresta ombrófila densa, em que os incêndios florestais não eram comuns (BARLOW et al. 2016), algumas espécies podem ser resilientes ao estresse causado pelo fogo.

Os resultados indicam que Rinorea guianensis e Protium apiculatum, são competidoras de extrema importância na comunidade, pois, embora tenham reduzido suas populações, estas se mantiveram, tanto em T0 quanto em T1, como as espécies com maior densidade (árvore ha-1), durante os 29 anos. Além destas espécies, vale destacar, também, Cordia spp., Cecropia sciadophylla e Aparasthmium cordatum que, após o fogo, apresentaram crescimento de suas populações que as colocou em posição de destaque na comunidade florestal. Reis et at. (2010) ao avaliarem o potencial madeireiro de uma área, após 29 anos da primeira exploração florestal, destacaram alguns gêneros com alta abundância, dentre eles Protium e Rinorea. Além disso, os autores registraram altas volumetrias para Bixa arborea, Inga spp., Rinorea guianensis e Virola michelli demonstrando a capacidade destas espécies de se beneficiar das clareiras abertas por distúrbios (extração seletiva de madeira e/ou incêndios, por exemplo).

Tabela 1 - Parâmetros estruturais das 10 espécies com maiores valores de Índice de Valor de Importância (IVI) nas medições anteriores (1983 e 1995) e posteriores ao incêndio (2008 e 2012) em uma área de floresta ombrófila densa sem histórico de distúrbios (T0), na Floresta Nacional do Tapajós, Pará, Brasil

Table 1 - Structural parameters of the 10 species with the highest values of Import Value Index (IVI) in previous (1983 and 1995) and post-fire measurements (2008 and 2012) in an area of dense ombrophilous forest unaffected by fire (T0), in Tapajós National Forest, Pará state, Brazil

\begin{tabular}{|c|c|c|c|c|c|c|c|c|}
\hline \multicolumn{9}{|c|}{ T0 - 1983} \\
\hline Espécies & Família & FA & DA & DoA & FR & DoR & DR & IVI \\
\hline Rinorea guianensis Aubl & Violaceae & 6 & 124 & 9,86 & 0,01 & 0,07 & 0,04 & 0,04 \\
\hline $\begin{array}{l}\text { Manilkara elata (Allemão ex } \\
\text { Miq.) Monach. }\end{array}$ & Sapotaceae & 7 & 27 & 18,55 & 0,01 & 0,01 & 0,08 & 0,04 \\
\hline Protium apiculatum Swart & Burseraceae & 7 & 89 & 4,12 & 0,01 & 0,05 & 0,02 & 0,03 \\
\hline $\begin{array}{l}\text { Tachigali chrysophylla (Poepp.) } \\
\text { Zarucchi \& Herend. }\end{array}$ & Fabaceae & 7 & 42 & 8,31 & 0,01 & 0,02 & 0,04 & 0,02 \\
\hline $\begin{array}{l}\text { Eschweilera coriacea (DC.) } \\
\text { S.A.Mori }\end{array}$ & Lecythidaceae & 7 & 54 & 5,81 & 0,01 & 0,03 & 0,03 & 0,02 \\
\hline Duguetia echinophora R.E.Fr. & Annonaceae & 7 & 74 & 3,15 & 0,01 & 0,04 & 0,01 & 0,02 \\
\hline Inga spp. & Fabaceae & 7 & 72 & 2,75 & 0,01 & 0,04 & 0,01 & 0,02 \\
\hline Geissospermum sericeum Miers & Apocynaceae & 6 & 22 & 6,68 & 0,01 & 0,01 & 0,03 & 0,02 \\
\hline Tachigali glauca Tul. & Fabaceae & 7 & 26 & 4,55 & 0,01 & 0,01 & 0,02 & 0,01 \\
\hline Amphiodon effusus Huber & Fabaceae & 6 & 53 & 1,37 & 0,01 & 0,03 & 0,01 & 0,01 \\
\hline Subtotal & & 67 & 583 & 65,16 & 0,10 & 0,32 & 0,29 & 0,24 \\
\hline Outras & & 631 & 1238 & 158,21 & 0,90 & 0,68 & 0,71 & 0,76 \\
\hline Total & & 698 & 1821 & 223 & 1 & 1 & 1 & 1 \\
\hline
\end{tabular}


Tabela 1 - Continuação ...

Table 1 - Continuation ...

\begin{tabular}{|c|c|c|c|c|c|c|c|c|}
\hline \multicolumn{9}{|c|}{ T0 - 1995} \\
\hline Espécies & Família & FA & DA & DoA & FR & DoR & DR & IVI \\
\hline Rinorea guianensis Aubl & Violaceae & 6 & 120 & 10,31 & 0,01 & 0,06 & 0,05 & 0,04 \\
\hline $\begin{array}{l}\text { Manilkara elata (Allemão ex } \\
\text { Miq.) Monach. }\end{array}$ & Sapotaceae & 7 & 23 & 19,55 & 0,01 & 0,01 & 0,09 & 0,04 \\
\hline Protium apiculatum Swart & Burseraceae & 7 & 92 & 4,54 & 0,01 & 0,05 & 0,02 & 0,03 \\
\hline $\begin{array}{l}\text { Tachigali chrysophylla (Poepp.) } \\
\text { Zarucchi \& Herend. }\end{array}$ & Fabaceae & 7 & 48 & 9,56 & 0,01 & 0,03 & 0,04 & 0,03 \\
\hline $\begin{array}{l}\text { Eschweilera coriacea (DC.) } \\
\text { S.A.Mori }\end{array}$ & Lecythidaceae & 7 & 53 & 6,41 & 0,01 & 0,03 & 0,03 & 0,02 \\
\hline Duguetia echinophora R.E.Fr. & Annonaceae & 7 & 72 & 3,50 & 0,01 & 0,04 & 0,02 & 0,02 \\
\hline Inga spp. & Fabaceae & 7 & 62 & 2,65 & 0,01 & 0,03 & 0,01 & 0,02 \\
\hline Geissospermum sericeum Miers & Apocynaceae & 6 & 22 & 7,24 & 0,01 & 0,01 & 0,03 & 0,02 \\
\hline $\begin{array}{l}\text { Onychopetalum amazonicum } \\
\text { R.E.Fr. }\end{array}$ & Annonaceae & 7 & 53 & 1,41 & 0,01 & 0,03 & 0,01 & 0,01 \\
\hline Carapa guianensis Aubl. & Meliaceae & 5 & 20 & 5,95 & 0,01 & 0,01 & 0,03 & 0,01 \\
\hline Subtotal & & 66 & 565 & 71,14 & 0,09 & 0,30 & 0,32 & 0,24 \\
\hline Outras & & 655 & 1293 & 151,98 & 0,91 & 0,70 & 0,68 & 0,76 \\
\hline Total & & 721 & 1858 & 223 & 1 & 1 & 1 & 1 \\
\hline
\end{tabular}

T0 - 2008

\begin{tabular}{|c|c|c|c|c|c|c|c|c|}
\hline Espécies & Famíliia & FA & DA & DoA & FR & DoR & DR & IVI \\
\hline Rinorea guianensis Aubl & Violaceae & 6 & 122 & 11,38 & 0,01 & 0,07 & 0,05 & 0,04 \\
\hline $\begin{array}{l}\text { Manilkara elata (Allemão ex } \\
\text { Miq.) Monach. }\end{array}$ & Sapotaceae & 6 & 21 & 20,37 & 0,01 & 0,01 & 0,08 & 0,03 \\
\hline $\begin{array}{l}\text { Tachigali chrysophylla (Poepp.) } \\
\text { Zarucchi \& Herend. }\end{array}$ & Fabaceae & 7 & 40 & 13,16 & 0,01 & 0,02 & 0,05 & 0,03 \\
\hline Protium apiculatum Swart & Burseraceae & 7 & 89 & 4,96 & 0,01 & 0,05 & 0,02 & 0,03 \\
\hline $\begin{array}{l}\text { Eschweilera coriacea (DC.) } \\
\text { S.A.Mori }\end{array}$ & Lecythidaceae & 7 & 45 & 7,37 & 0,01 & 0,02 & 0,03 & 0,02 \\
\hline $\begin{array}{l}\text { Onychopetalum amazonicum } \\
\text { R.E.Fr. }\end{array}$ & Annonaceae & 7 & 67 & 1,86 & 0,01 & 0,04 & 0,01 & 0,02 \\
\hline Duguetia echinophora R.E.Fr. & Annonaceae & 7 & 54 & 3,05 & 0,01 & 0,03 & 0,01 & 0,02 \\
\hline Geissospermum sericeum Miers & Apocynaceae & 6 & 20 & 7,22 & 0,01 & 0,01 & 0,03 & 0,02 \\
\hline Carapa guianensis Aubl. & Meliaceae & 5 & 19 & 5,64 & 0,01 & 0,01 & 0,02 & 0,01 \\
\hline Inga spp. & Fabaceae & 7 & 43 & 1,64 & 0,01 & 0,02 & 0,01 & 0,01 \\
\hline Subtotal & & 65 & 520 & 76,64 & 0,09 & 0,28 & 0,32 & 0,23 \\
\hline Outras & & 655 & 1306 & 165,70 & 0,91 & 0,72 & 0,68 & 0,77 \\
\hline Total & & 720 & 1826 & 242 & 1 & 1 & 1 & 1 \\
\hline
\end{tabular}


Tabela 1 - Conclusão ...

Table 1 - Conclusion ...

\begin{tabular}{|c|c|c|c|c|c|c|c|c|}
\hline \multicolumn{9}{|c|}{ T0 - 2012} \\
\hline Espécies & Família & FA & DA & DoA & FR & DoR & DR & IVI \\
\hline Rinorea guianensis Aubl & Violaceae & 6 & 123 & 11,72 & 0,01 & 0,07 & 0,05 & 0,04 \\
\hline $\begin{array}{l}\text { Manilkara elata (Allemão ex } \\
\text { Miq.) Monach. }\end{array}$ & Sapotaceae & 6 & 20 & 20,73 & 0,01 & 0,01 & 0,09 & 0,03 \\
\hline $\begin{array}{l}\text { Tachigali chrysophylla (Poepp.) } \\
\text { Zarucchi \& Herend. }\end{array}$ & Fabaceae & 7 & 40 & 14,51 & 0,01 & 0,02 & 0,06 & 0,03 \\
\hline Protium apiculatum Swart & Burseraceae & 7 & 90 & 5,33 & 0,01 & 0,05 & 0,02 & 0,03 \\
\hline $\begin{array}{l}\text { Eschweilera coriacea (DC.) } \\
\text { S.A.Mori }\end{array}$ & Lecythidaceae & 7 & 42 & 7,82 & 0,01 & 0,02 & 0,03 & 0,02 \\
\hline $\begin{array}{l}\text { Onychopetalum amazonicum } \\
\text { R.E.Fr. }\end{array}$ & Annonaceae & 7 & 68 & 1,88 & 0,01 & 0,04 & 0,01 & 0,02 \\
\hline Geissospermum sericeum Miers & Apocynaceae & 6 & 20 & 7,32 & 0,01 & 0,01 & 0,03 & 0,02 \\
\hline Duguetia echinophora R.E.Fr. & Annonaceae & 7 & 50 & 2,74 & 0,01 & 0,03 & 0,01 & 0,02 \\
\hline Carapa guianensis Aubl. & Meliaceae & 6 & 19 & 5,92 & 0,01 & 0,01 & 0,02 & 0,01 \\
\hline $\begin{array}{l}\text { Maquira sclerophylla (Ducke) } \\
\text { C.C.Berg. }\end{array}$ & Moraceae & 7 & 37 & 2,07 & 0,01 & 0,02 & 0,01 & 0,01 \\
\hline Subtotal & & 66 & 509 & 80,04 & 0,09 & 0,28 & 0,33 & 0,23 \\
\hline Outras & & 656 & 1318 & 161,89 & 0,91 & 0,72 & 0,67 & 0,77 \\
\hline Total & & 722 & 1827 & 241,92 & 1 & 1 & 1 & 1 \\
\hline
\end{tabular}

Fonte: Autores (2019)

Em que: $\mathrm{FA}=$ Frequência Absoluta, $\mathrm{DA}=$ Densidade Absoluta, DoA $=$ Dominância Absoluta, $\mathrm{FR}=$ Frequência relativa, $\mathrm{DoR}=$ Dominância relativa, DR = Densidade Relativa, IVI = Índice de Valor de Importância.

Tabela 2 - Parâmetros estruturais das 10 espécies com maiores valores de Índice de Valor de Importância (IVI) nas medições anteriores (1983 e 1995) e posteriores ao incêndio (2008 e 2012) em uma área de floresta ombrófila densa atingida por fogo (T1), na Floresta Nacional do Tapajós, Pará, Brasil

Table 2 - Structural parameters of the 10 species with highest values of Import Value Index (IVI) in previous (1983 and 1995) and post-fire measurements (2008 and 2012) in an area of dense ombrophilous forest affected by fire (T1), in Tapajós National Forest, Pará state, Brazil

\begin{tabular}{lccccccccc}
\hline \multicolumn{1}{c}{ ANTES DO FOGO } \\
\hline \multicolumn{1}{c}{ T1 - 1983 } \\
\hline \multicolumn{1}{c}{ Espécies } & Família & FA & DA & DoA & FR & DoR & DR & IVI \\
\hline Rinorea guianensis Aubl & Violaceae & 5 & 103 & 7,12 & 0,01 & 0,07 & 0,05 & 0,04 \\
Geissospermum sericeum Miers & Apocynaceae & 5 & 25 & 10,22 & 0,01 & 0,02 & 0,08 & 0,03 \\
Inga spp. & Fabaceae & 5 & 80 & 4,77 & 0,01 & 0,05 & 0,04 & 0,03
\end{tabular}


Tabela 2 - Continuação ...

Table 2 - Continuation ...

\begin{tabular}{|c|c|c|c|c|c|c|c|c|}
\hline \multicolumn{9}{|c|}{ ANTES DO FOGO } \\
\hline \multicolumn{9}{|c|}{ T1 - 1983} \\
\hline Espécies & Família & FA & DA & DoA & FR & DoR & DR & IVI \\
\hline Protium apiculatum Swart & Burseraceae & 5 & 81 & 3,57 & 0,01 & 0,05 & 0,03 & 0,03 \\
\hline $\begin{array}{l}\text { Eschweilera coriacea (DC.) } \\
\text { S.A.Mori }\end{array}$ & Lecythidaceae & 5 & 46 & 5,16 & 0,01 & 0,03 & 0,04 & 0,03 \\
\hline Não identificada & Sapotaceae & 5 & 37 & 3,93 & 0,01 & 0,02 & 0,03 & 0,02 \\
\hline $\begin{array}{l}\text { Iryanthera sagotiana (Benth.) } \\
\text { Warb. }\end{array}$ & Myristicaceae & 5 & 16 & 4,76 & 0,01 & 0,01 & 0,03 & 0,02 \\
\hline $\begin{array}{l}\text { Maquira sclerophylla (Ducke) } \\
\text { C.C.Berg. }\end{array}$ & Moraceae & 5 & 35 & 3,01 & 0,01 & 0,02 & 0,02 & 0,02 \\
\hline Neea floribunda Poepp. \& Endl. & Nyctaginaceae & 5 & 38 & 2,44 & 0,01 & 0,02 & 0,02 & 0,02 \\
\hline Duguetia echinophora R.E.Fr. & Annonaceae & 5 & 42 & 1,47 & 0,01 & 0,03 & 0,01 & 0,02 \\
\hline Subtotal & & 50 & 503 & 46,45 & 0,09 & 0,33 & 0,34 & 0,25 \\
\hline Outras & & 528 & 1034 & 89,66 & 0,91 & 0,67 & 0,66 & 0,75 \\
\hline Total & & 578 & 1537 & 136,10 & 1 & 1 & 1 & 1 \\
\hline \multicolumn{9}{|c|}{ T1 - 1995} \\
\hline Espécies & Família & FA & DA & DoA & FR & DoR & DR & IVI \\
\hline Rinorea guianensis Aubl & Violaceae & 5 & 106 & 7,79 & 0,01 & 0,07 & 0,05 & 0,04 \\
\hline Geissospermum sericeum Miers & Apocynaceae & 5 & 28 & 10,70 & 0,01 & 0,02 & 0,07 & 0,03 \\
\hline Protium apiculatum Swart. & Burseraceae & 5 & 88 & 4,07 & 0,01 & 0,06 & 0,03 & 0,03 \\
\hline Inga spp. & Fabaceae & 5 & 67 & 3,58 & 0,01 & 0,04 & 0,02 & 0,03 \\
\hline $\begin{array}{l}\text { Eschweilera coriacea (DC.) } \\
\text { S.A.Mori }\end{array}$ & Lecythidaceae & 5 & 42 & 4,80 & 0,01 & 0,03 & 0,03 & 0,02 \\
\hline Não identificada & Sapotaceae & 5 & 32 & 3,78 & 0,01 & 0,02 & 0,03 & 0,02 \\
\hline $\begin{array}{l}\text { Maquira sclerophylla (Ducke) } \\
\text { C.C.Berg. }\end{array}$ & Moraceae & 5 & 35 & 3,27 & 0,01 & 0,02 & 0,02 & 0,02 \\
\hline Neea floribunda Poepp. \& Endl. & Nyctaginaceae & 5 & 35 & 2,66 & 0,01 & 0,02 & 0,02 & 0,02 \\
\hline Swartzia polyphylla DC. & Fabaceae & 2 & 2 & 6,48 & 0,00 & 0,00 & 0,04 & 0,02 \\
\hline Erisma uncinatum Warm. & Vochysiaceae & 3 & 3 & 4,84 & 0,01 & 0,00 & 0,03 & 0,01 \\
\hline Subtotal & & 45 & 438 & 52 & 0,08 & 0,29 & 0,36 & 0,24 \\
\hline Outras & & 545 & 1064 & 92,43 & 0,92 & 0,71 & 0,64 & 0,76 \\
\hline Total & & 590 & 1502 & 144 & 1 & 1 & 1 & 1 \\
\hline
\end{tabular}


Tabela 2 - Conclusão ...

Table 2 - Conclusion ...

\begin{tabular}{|c|c|c|c|c|c|c|c|c|}
\hline \multicolumn{9}{|c|}{ PÓS- FOGO } \\
\hline \multicolumn{9}{|c|}{ T1 - 2008} \\
\hline Espécies & Família & FA & DA & DoA & FR & DoR & DR & IVI \\
\hline Rinorea guianensis Aubl. & Violaceae & 5 & 86 & 7,20 & 0,01 & 0,06 & 0,05 & 0,04 \\
\hline Geissospermum sericeum Miers & Apocynaceae & 5 & 30 & 11,88 & 0,01 & 0,02 & 0,08 & 0,04 \\
\hline Protium apiculatum Swart & Burseraceae & 5 & 61 & 2,83 & 0,01 & 0,04 & 0,02 & 0,02 \\
\hline $\begin{array}{l}\text { Eschweilera coriacea (DC.) } \\
\text { S.A.Mori }\end{array}$ & Lecythidaceae & 5 & 25 & 3,55 & 0,01 & 0,02 & 0,02 & 0,02 \\
\hline Neea floribunda Poepp. \& Endl. & Nyctaginaceae & 5 & 30 & 2,71 & 0,01 & 0,02 & 0,02 & 0,02 \\
\hline $\begin{array}{l}\text { Tachigali chrysophylla (Poepp.) } \\
\text { Zarucchi \& Herend. }\end{array}$ & Fabaceae & 5 & 11 & 4,58 & 0,01 & 0,01 & 0,03 & 0,02 \\
\hline Swartzia polyphylla DC. & Fabaceae & 1 & 1 & 6,51 & 0,00 & 0,00 & 0,04 & 0,02 \\
\hline Erisma uncinatum Warm. & Vochysiaceae & 2 & 2 & 6,03 & 0,00 & 0,00 & 0,04 & 0,02 \\
\hline Inga spp. & Fabaceae & 5 & 33 & 1,82 & 0,01 & 0,02 & 0,01 & 0,02 \\
\hline $\begin{array}{l}\text { Maquira sclerophylla (Ducke) } \\
\text { C.C.Berg. }\end{array}$ & Moraceae & 5 & 22 & 2,88 & 0,01 & 0,02 & 0,02 & 0,01 \\
\hline Subtotal & & 43 & 301 & 50 & 0,07 & 0,22 & 0,34 & 0,21 \\
\hline Outras & & 539 & 1066 & 96,72 & 0,93 & 0,78 & 0,66 & 0,79 \\
\hline Total & & 582 & 1367 & 146,72 & 1 & 1 & 1 & 1 \\
\hline \multicolumn{9}{|c|}{ T1 - 2012} \\
\hline Espécies & Família & FA & DA & DoA & FR & DoR & DR & IVI \\
\hline Rinorea guianensis Aubl & Violaceae & 5 & 84 & 7,14 & 0,01 & 0,06 & 0,05 & 0,04 \\
\hline Geissospermum sericeum Miers & Apocynaceae & 5 & 30 & 12,08 & 0,01 & 0,02 & 0,08 & 0,04 \\
\hline Protium apiculatum Swart & Burseraceae & 5 & 63 & 2,97 & 0,01 & 0,04 & 0,02 & 0,02 \\
\hline $\begin{array}{l}\text { Tachigali chrysophylla (Poepp.) } \\
\text { Zarucchi \& Herend. }\end{array}$ & Fabaceae & 5 & 14 & 6,07 & 0,01 & 0,01 & 0,04 & 0,02 \\
\hline Neea floribunda Poepp. \& Endl. & Nyctaginaceae & 5 & 30 & 2,83 & 0,01 & 0,02 & 0,02 & 0,02 \\
\hline $\begin{array}{l}\text { Maquira sclerophylla (Ducke) } \\
\text { C.C.Berg. }\end{array}$ & Moraceae & 5 & 25 & 3,14 & 0,01 & 0,02 & 0,02 & 0,02 \\
\hline Erisma uncinatum Warm. & Vochysiaceae & 2 & 2 & 6,30 & 0,00 & 0,00 & 0,04 & 0,02 \\
\hline Swartzia polyphylla DC. & Fabaceae & 1 & 1 & 6,51 & 0,00 & 0,00 & 0,04 & 0,02 \\
\hline Couratari stellata A.C.Sm. & Lecythidaceae & 5 & 12 & 3,91 & 0,01 & 0,01 & 0,03 & 0,01 \\
\hline Cordia spp. & Boraginaceae & 5 & 38 & 1,13 & 0,01 & 0,03 & 0,01 & 0,01 \\
\hline Subtotal & & 43 & 299 & 52,08 & 0,07 & 0,21 & 0,34 & 0,21 \\
\hline Outras & & 551 & 1140 & 99,66 & 0,93 & 0,79 & 0,66 & 0,79 \\
\hline Total & & 594 & 1439 & 152 & 1 & 1 & 1 & 1 \\
\hline
\end{tabular}

Fonte: Autores (2019)

Em que: $\mathrm{FA}=$ Frequência Absoluta, $\mathrm{DA}=$ Densidade Absoluta, DoA $=$ Dominância Absoluta, $\mathrm{FR}=$ Frequência relativa, $\mathrm{DoR}$ = Dominância relativa, $\mathrm{DR}=$ Densidade Relativa, IVI = Índice de Valor de Importância. 


\section{Conclusões}

Após 15 anos da ocorrência do incêndio, a floresta não apresentou prejuízos na riqueza, estrutura e diversidade de espécies. As alterações pós-fogo na composição florística de uma floresta primária na Amazônia são definidas, principalmente, pela entrada e saída de espécies localmente raras, e as espécies com maiores abundâncias e área basal são responsáveis pela manutenção da estrutura da floresta, destacando-se dentre elas: Rinorea guianensis Aubl. e Protium apiculatum Swart.

\section{Agradecimentos}

Agradecemos à EMBRAPA Amazônia Oriental por disponibilizar os dados de monitoramento das parcelas permanentes. Agradecemos, também, ao ICMBio por apoiar e incentivar a capacitação de seus servidores e a realização de pesquisa científica nas Unidades de Conservação Federais.

\section{Referências}

AMARAL, D. D. et al. Checklist da flora arbórea de remanescentes florestais da região metropolitana de Belém, Pará, Brasil. Boletim do Museu Paraense Emilio Goeldi, Ciências Naturais, Belém, v. 4, n. 3, p. 231-289, 2009.

ANDRADE, D. F. C. de et al. Post-fire recovery of a dense ombrophylous forest in Amazon. Anais da Academia Brasileira de Ciências, Rio de Janeiro, v. 91, n. 2, e20170840, 2019.

ANDRADE, D. F. C. de, GAMA, J. R.V., MELO, L. O., RUSCHEL, A. R. Inventário florestal de grandes áreas na Floresta Nacional do Tapajós, Pará, Amazônia, Brasil. Biota Amazônia, Macapá, v. 5, n. 1, p. 109-115, 2015.

APG IV. An update of the Angiosperm Phylogeny Group classification for the orders and families of flowering plants: APG IV. Botanical Journal of the Linnean Society, London, v. 181, p. 1-20, 2016

ARAUJO, H. J. B. de et al. Danos provocados pelo fogo sobre a vegetação natural em uma floresta primária no estado do Acre, Amazônia Brasileira. Ciência Florestal, Santa Maria, v. 23, n. 2, p. 297-308, 2013.

AVILA, A. L. de et al. Medium-term dynamics of tree species composition in response to silvicultural intervention intensities in a tropical rain forest. Biological Conservation, Boston, v. 191, p. 577-586, 2015.

BARLOW J. et al. Anthropogenic disturbance in tropical forests can double biodiversity loss from deforestation. Nature, London, v. 535, p. 144-147, 2016.

BARLOW, J. et al. Ecological responses to el Niño-induced surface fires in central Brazilian Amazonia: management implications for flammable tropical forests. Philosophical transactions of the Royal Society B, Biological sciences, London, v. 359, n. 1443, p. 367-380, 2004.

CARVALHO, J. O. P. de. Changes in the floristic composition of a terra firme rain forest in 
Brazilian Amazonia over an eight-year period in response to logging. Acta Amazonica, Manaus, v. 32, n. 2, p. 277-291, 2002.

CHAZDON, R. L. Regeneração de florestas tropicais. Boletim do Museu Paraense Emílio Goeldi, Ciências Naturais, Belém, v. 7, n. 3, p. 195-218, 2012.

COLWELL, R. K.; CODDINGTON, J. A. Estimating terrestrial biodiversity through extrapolation. Philosophical Transactions of the Royal Society (Series B), London, v. 345, 101-118. 1994.

CONDÉ, T. M.; TONINI, H. Fitossociologia de uma Floresta Ombrófila Densa na Amazônia Setentrional, Roraima, Brasil. Acta Amazonica, Manaus, v. 43, n. 3, p. 247-259, 2013.

COSTA, D. H. M.; DE CARVALHO, J. O. P.; SILVA, J. N. M. Dinâmica da composição florística após a colheita de madeira em uma área de terra firme na Floresta Nacional do Tapajós. Revista de Ciências Agrárias, Belém, n. 38, p. 67-90, 2002.

GONÇALVES, F. G.; SANTOS, J. R. dos. Composição florística e estrutura de uma unidade de manejo florestal sustentável na Floresta Nacional do Tapajós, Pará. Acta Amazonica, Manaus, v. 38, n. 2, p. 229-244, 2008.

HOLDSWORTH, A. R.; UHL, C. Fire in Amazonian selectively-logged rain forest and the potential for fire reduction. Ecological Applications, Washington, v. 7, n. 2, p. 713-725, 1997.

JARDIM, F. C. da S.; SOARES, M. da S. Comportamento de Sterculia pruriens (Aubl.) Schum. em floresta tropical manejada em Moju-PA. Acta Amazonica, Manaus, v. 40, n. 3, p. 535-542, 2010.

KARSTEN, R. J. et al. Regeneration in canopy gaps of tierra-firme forest in the Peruvian Amazon: Comparing reduced impact logging and natural, unmanaged forests. Forest Ecology and Management, Amsterdam, v. 310, p. 663-671, 2013.

LEGENDRE, P; LEGENDRE. Numerical Ecology with R, Third English Edition. Amsterdam: Elsevier, 2012. 990 p.

MARTINS, F da S. R. V.; XAUD, H. A. M.; SANTOS, J. R. dos; GALVÃO, L. S. Effects of fire on above-ground forest biomass in the northern Brazilian Amazon. Journal of Tropical Ecology, Amsterdam, v. 28, n. 6, p. 591-601, 2012.

MEGGERS, B. J. Archeological evidence for the impact of mega-Nino events on Amazonia during the past two millennia. Climatic Change, [s. 1.], v. 28, n. 4, p. 321-338, 1994.

OLIVEIRA JUNIOR, R. C. de et al. Chemical analysis of rainfall and throughfall in the Tapajós National Forest, Belterra, Pará, Brazil. An Interdisciplinary Journal of Applied Science, Taubaté, 10, 263-285, 2015.

OLIVEIRA, A. N. de; AMARAL, I. L. do. Florística e fitossociologia de uma floresta de vertente na Amazônia Central, Amazonas, Brasil. Acta Amazonica, Manaus, v. 34, n. 1, p. 21-34, 2004.

OLIVEIRA, L. C. de et al. Efeito da exploração de madeira e tratamentos silviculturais na composição florística e diversidade de espécies em uma área de 136 ha na Floresta Nacional do Tapajós, Belterra. Scientia Forestalis, Piracicaba, v. 69, n. 3, p. 62-76, 2005.

QUARESMA, A. C. et al. Composition, diversity and structure of vascular epiphytes in two contrasting Central Amazonian flood plain ecosystems. Acta Botanica Brasilica, Belo Horizonte, v. 31, n. 4, p. 686-697, 2017.

REFLORA. Herbário Virtual. Jardim Botânico do Rio de Janeiro. Disponível em: reflora.jbrj. gov.br/reflora/herbarioVirtual/. Acesso em: 22 nov. 2018.

REIS, L. P. et al. Avaliação do potencial madeireiro na Floresta Nacional do Tapajós após 28 anos da exploração florestal. Pesquisa Florestal Brasileira, Colombo, v. 30, n. 64, p. 265-281, 2010.

REIS, S. M. et al. Post-fire dynamics of the woody vegetation of a savanna forest (Cerradão) in the 
Cerrado-Amazon transition zone. Acta Botanica Brasilica, Belo Horizonte, v. 29, n. 3, p. 408416, 2015.

SILVA, J. N. et al. Diretrizes para instalação e medição de parcelas permanentes em florestas naturais da Amazônia Brasileira. Belém, PA: EMBRAPA-CPATU, 2005. 36 p.

SILVA, K. E. da; MATOS, F. D. de A.; FERREIRA, M. M. Composição florística e fitossociologia de espécies arbóreas do Parque Fenológico da Embrapa Amazônia Ocidental. Acta Amazonica, Manaus, v. 38, n. 2, p. 213-222, 2008.

SIT, V.; TAYLOR, B. Land Management Handbook: Statistical methods for Adaptive Management Studies. Victoria: British Columbia Ministry of Forests Research Program, 1998. $148 \mathrm{p}$.

SOUZA, D. R. de et al. Análise estrutural em floresta ombrófila densa de terra firme não explorada, Amazônia Oriental. Revista Árvore, Viçosa, v. 30, n. 1 p. 75-87, 2006.

TER STEEGE, H. et al. Hyperdominance in the Amazonian tree flora. Science, Washington, v. 342, n. 6156, p. 1243092:1-9, 2013.

VON ENDE, C. N. Repeated-measures analysis: growth and other time-dependent measures. In: SCHEINER, S. M.; GUREVITCH, J. (Eds.). Design and analysis of ecological experiments. London: Chapman \& Hall, 1993. p. 113-137. 